\title{
INFLUENCE OF LONG-TERM CYTOTOXIC CHEMOTHERAPY ON THE CONDITION OF PERIPHERAL VENOUS CHANNEL
}

\author{
Tetiana Tkachuk \\ National Pirogov Memorial Medical University \\ 56 Pirogov str., Vinnytsya, Ukraine, 21018 \\ tvlad2103@gmail.com
}

\begin{abstract}
The necessity of long-term venous access in cancer patients appears at frequent and long-term courses of cytotoxic therapy. Peripheral veins of forearms are most often used for these aims. The conditions of peripheral venous channel in 32 cancer patients, who underwent the long-term treatment with antitumor preparations were analyzed in the article on own investigatory material.

The methods of dopplerography, morphological and immunohystochemical studies were used.

The qualitative and quantitative dopplerographic changes in forearm veins in different terms after chemotherapy start were revealed in most patients.

The conclusion was made about unsuitability of forearm peripheral veins for the long term administration of cytostatics and the necessity to create the alternative vascular access that would correspond to the criteria of safety, reliability and long-term exploitation.
\end{abstract}

Keywords: peripheral venous access, repeated administration of chemopreparations, results.

\section{Introduction}

According to the data of international cancer-registers of different countries, during last 50 years the improvement of cancer patients treatment results took place that was manifested by the increase of general relapse-free survival rate at the main cancer localizations [1]. It became possible not only due to the early diagnostics and realizations of the programs of populational screening but also at the expanse of intensification of antitumor therapy methods [2].

Today the effective treatment of cancer patient can be realized in radical, palliative and symptomatic regime and include the components of accompaniment theraphy. Practically all technologies of antitumor theraphy need creation of reliable long-term access to the patient's vascular system. The modern chemotherapy is not possible without taking into account the life quality of cancer patients [3].

The necessity of long-term access to blood of a cancer patient appears at frequent and longterm courses of cytotoxic therapy, biotherapy, transfusion of blood and its components, hydration, supporting therapy (total parenteral nutrition, system administration of antibiotics or colony-stimulating factors), methods of efferent therapy (different methods of cytopheresis or reinfusion of marrow at its autotransplantation), pain therapy and other methods of radical, palliative and symptomatic treatment [4] .

Today the external catheters of different construction, placed in peripheral or central veins and also "fully implanted vascular systems" are used for these aims most often [5].

The one of most important conditions of chemotherapy conduct is a possibility of regular, long-term and safe administration of cytostatic preparations [6]. It means that if synthetic intravascular prostheses are used as a vascular access, they must be biologically inert, at that the risk of infectious and thrombotic complications development at setting and exploitation of the system must be minimal [4].

The long-term character provides such duration of access use that is necessary for realization of whole planned program of cancer patients' treatment without the necessity of formation of a new access [7].

The repeated punctures of peripheral veins at administration of antitumor preparations are associated with development of the whole series of serious complications. Most often there are observed the mechanical lesion of venous wall and obliteration of vein lumen, development of chem- 
ical phlebitis, phlebothrombosis, functional defect of venous channel of extremity at extravasation of antitumor preparation [8].

The important role in development of chemical phlebitis and thrombosis of peripheral veins is also played by endothelial dysfunction [9].

The spectrum of vascular complications, appeared on the background of chemotherapeutic preparations use, is very wide: from asymptomatic phlebitis to potentially lethal vasculopathies (vein-occlusive disease and thrombotic microangiopathy) [10].

The lesion and proliferation of endothelium can develop both at peak concentrations of cytostatic at its administration and at low ones of antitumor preparations.

The choice of central vein (subclavian, internal jugular or femoral one) for setting catheter has a great importance. Today a jugular vein is preferred. Stenosis often develops at using subclavian vein that results in chronic venous hypertension of upper extremity [11]. The disorder of venous outflow influences not only the development of forearm tissues edema but also can be the cause of upper extremity veins thrombosis [12].

After catheterization of subluvian vein, stenosis appears in $50 \%$ of patients. This complication is a risk factor of vascular access infection in $66,6 \%$ of patients against $33,3 \%$ of ones with normal venous outflow. The authors consider mechanical irritation of endothelial vein and infection as the causes of stenosis [13].

Exploitation of central venous catheters in cancer patients can be limited to the different clinical situations (planning of surgical interventions on neck or thorax, necessity of radiotherapy in this zone, presence of tumors in "head-neck" zone, in mediastinum, in mammary gland, lymph nodes of armpit zone, compression and stenosis of upper cava).

There are potentially dangerous complications, connected with both setting and exploitation of such catheters, occurred with different frequency, such as pneumothorax, iatrogenic puncture of artery, hemothorax as a result of large vessel lesion, local hematoma, incorrect placement of catheter, its damage (internal or external part), venous thrombosis, air embolism, extravasation of preparations, catheter-associated infection (sepsis) [14].

Granulocytopenia, (risk of sepsis), thrombocytopenia and coagulopathy (risk of bleeding) and also active infection must be considered as the absolute contraindications to the setting of longterm central venous access [15].

Today the completely implanted venous devices provide the comfortable access for intravascular administration of chemotherapeutic preparations, ionic solutions, blood preparations and solutions for parenteral nutrition.

Nevertheless, in literature there are numerous descriptions of severe complications, appeared at both intravenous port implantation (accidental puncture and artery perforation, bleeding hematoma, air embolism, pneumothorax) and at long-term exploitation of the system (infectious complications, thrombosis of upper cava system, perforation of vein wall by catheter, fracture of catheter). The information about extravasation of chemotherapeutic preparations is published more and more often [16].

Clinical manifestations cytostatics extravasation can vary from inflammatory reaction, pain, edema, temperature rise, erythema, phlebitis, sclerosis and pigmentation in veins zone to chemical cellulite, induration, desquamation of skin, bubbles, up to severe lesion of tissues and necrosis [17].

Cytostatics extravasation can lead not also to the limitation of extremity function, worsening of life quality but also to the development of septic condition and as a result - to disorder of antitumor therapy protocols [18].

High peak concentrations of cytostatics at chemotherapy and also the chronic chemically induced activation of venous endothelium leads to its sclerosis [19].

At the same time at conducting chemotherapy the numerous intakes of venous blood are needed for the control of treatment toxicity and following of disease dynamics and also intravenous infusion of supporting therapy. With time these complications lead to significant technical difficulties in adequate venous access, make impossible the long-term use of upper extremities veins 
for cytostatics administration, limitation of possibilities of further treatment, disorder of antitumor therapy protocols, decrease of treatment effectiveness and, as a result, to tumor progression.

\section{Aim of research}

To study the functional condition of peripheral venous channel of upper extremities in cancer patients, who receive the long-term cytotoxic chemotherapy through a cubital vein.

\section{Materials and methods}

The work is based on the analysis of the results of examination, treatment and observation of 32 patients (18 men and 14 women) with histologically confirmed diagnosis of malignant neoplasm, who were on treatment in Vinnitsya and Zaporozhye cancer dispensaries during 2012-2015.

In all patients was the clinical necessity of the long-term cytotoxic antitumor chemotherapy by multiple courses. The courses of chemotherapy, exceeded 6 month, were considered as longterm ones. Chemotherapy was carried out in adjuvant and palliative regimes.

Cytotoxic chemotherapy was carried out through the temporal access in peripheral vein, realized by its puncture or catheterization by "butterfly" catheter.

The dopplerographic examination was carried out on the apparatus Lgi PVR/APL (USA) using sensor 7,2 $\mathrm{mHz}$. Three dopplerography regimes were used. The areas with abnormal bloodstream were searched using color Doppler mapping, based on direction analysis (CDM) or flow energy (CDME). The speed and direction of flow in the studied blood object were assessed by dopplersonography of vessels in impulse regime. The high-speed flows were studied using dopplersonography of vessels in permanently wavy regime. Siemens.

The duplex scanning of forearm veins was carried out on the apparatus Acuson $\times 300$ by

The examination of patients was carried out in lying position on spine. The "gel pillow" was obligatory used, sensor was kept above skin, because even little pressure on forearm veins was enough to reduce bloodstream in them.

At the beginning of study, the scanning was carried out in plane, perpendicular to anatomic course of vein to specify the vessels topography.

At transversal scanning the mutual positions of vessels, their diameter, thickness and density of walls and also the condition of perivasal tissues were determined. The transversal scanning allowed to determine the area of effective cross-section of vein, and scanning along the studied segment of vessel allowed to detect the areas of its stricture formation and to get the estimate parameters of stenosis.

At longitudinal scanning of the course, diameter, internal contour and density of walls of forearm veins, their elasticity and lumen condition were estimated. The thickness of media-intima complex was also measured by the distant wall. The Doppler study was carried out in several areas, transferring the sensor along the tissues planes and inspecting the area of venous channel, as large as possible.

The character of dopplerographic curve was studied and the distance from the beginning of anacrotic cog to the beginning of reflected dicrotic wave was estimated, the interval from the beginning of anacrotic rise of arterial pulse to the beginning of dicrotic wave, the height of reflected wave and the level of its appearance were studied.

The following quantitative parameters were used as dopplerography ones for assessment of forearm veins suitability for exploitation in dynamics: area of effective vessel cut (AEVC, mm), index of stricture formation (IS, \%), linear speed of bloodstream (TAmx1, cm/s) and volumetric speed of bloodstream (FV1, 1/min). V. basilica was used for bloodstream assessment.

\section{Research results}

The qualitative dopplerographic changes in forearm veins in different terms after beginning of therapy were detected in most examined patients $(26-81,3 \%)$.

The contour of walls of forearm veins, their lumen and valves condition at the beginning of treatment of cancer patients did not differ from the ones in general population of healthy people. 
The decrease of venous channel capacity of both forearms as a variant of anatomic norm was observed in 16 patients $(50,0 \%)$. In these patients the single access to the venous system was usually the short cubital vein.

At exploitation, most often after 3-4 chemotherapy courses there was observed the thickening of forearm vein wall not only in the place of its puncture but also on significant extent in the direction to cephalic vein. The decrease of elasticity of vein walls and diminution of its lumen diameter were synchronously registered. The internal contour was uneven, bumpy. The valvular apparatus of veins was practically destructed. The organized hematomas with calcification were observed in the places of punctures and paravasaly.

At the study of veins and soft tissues of forearms using ultrasonography and dopplerography the typical changes were observed - thickening and sclerosis of venous wall, narrowing of vein lumen to slit-like one (1-2 $\mathrm{mm}$ in diameter), and also paravasal proliferation of connective tissue (fibrosis). The specific changes of veins and tissues of forearm after 4-6 chemotherapy courses are presented on the Fig. 1-3.

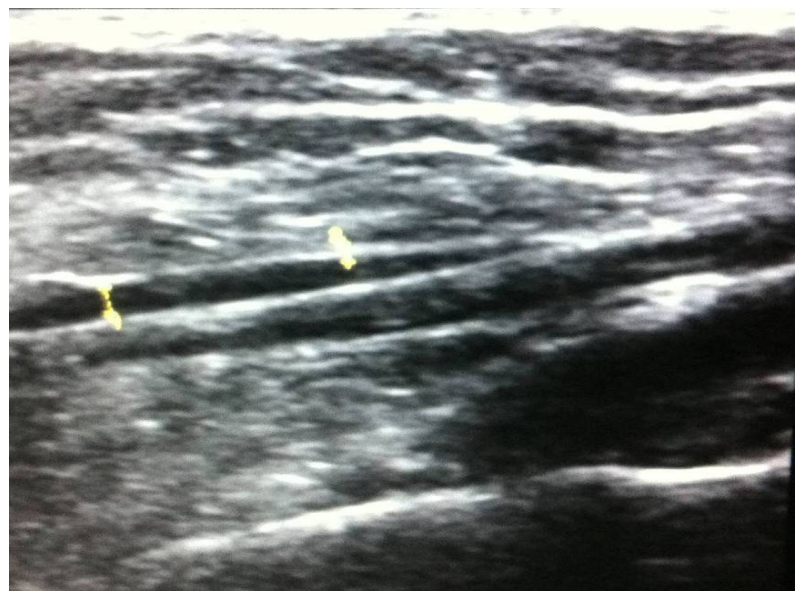

Fig. 1. Ultrasonography of veins and soft tissues of forearms in patient with chemical phlebitis after repeated administration of 5-phtoruracil. Sclerosis of venous wall is determined, but vein lumen is kept

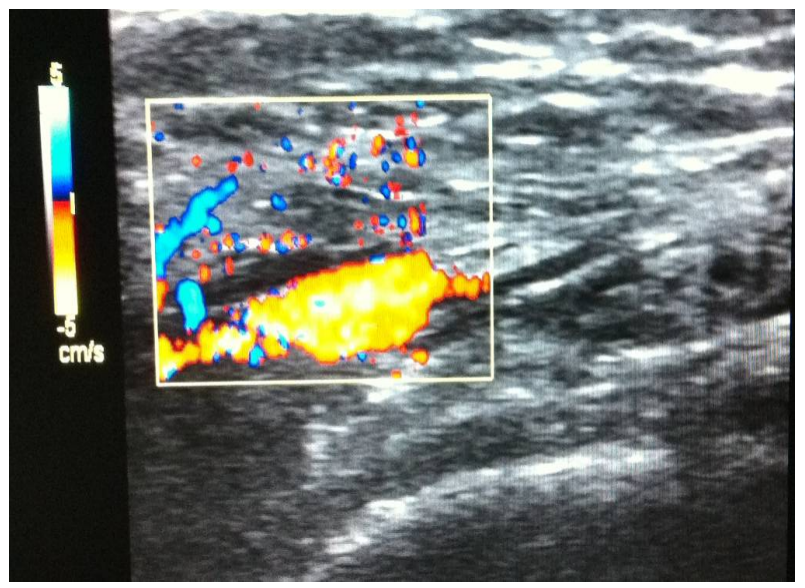

Fig. 2. Dopplerography of forearm veins, done after repeated administration of 5 -phtoruracil. Bloodstream in vein is kept, but abruptly decreased $(5-10 \mathrm{ml} / \mathrm{min})$.

Little forming collaterals are determined

The clinically significant acute complications at exploitation of forearm veins for polychemotherapy (extravasation) appeared in 4 patients (12,5\%). They were manifested by bubbles and tissues necrosis in the place of injection. In all observations the injured extremity could not be used 
for exploitation. For realization of treatment protocols at complications development, the temporal access to the central vein was set for further cytostatics administration.

The sonogram of forearm tissues at extravasation development is presented on the Fig. 4.

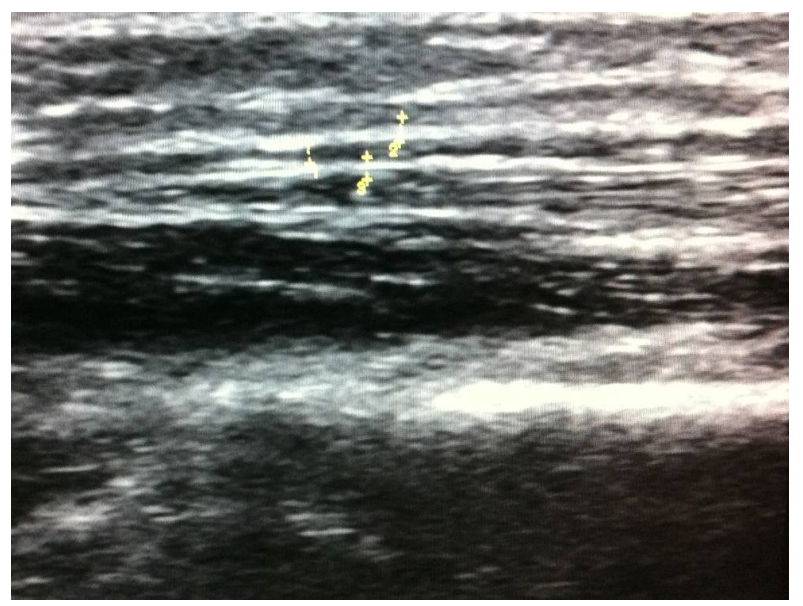

Fig. 3. Ultrasonography of veins and soft tissues of forearm in patient with chemical cellulite and induration of tissues. The chronic lesion developed after 6 chemotherapy courses by the scheme FOLFOX 4. The narrowing of vein lumen to $1-2 \mathrm{~mm}$ in diameter, thickening (sclerosis) of venous wall and paravasal fibrosis of tissues was determined

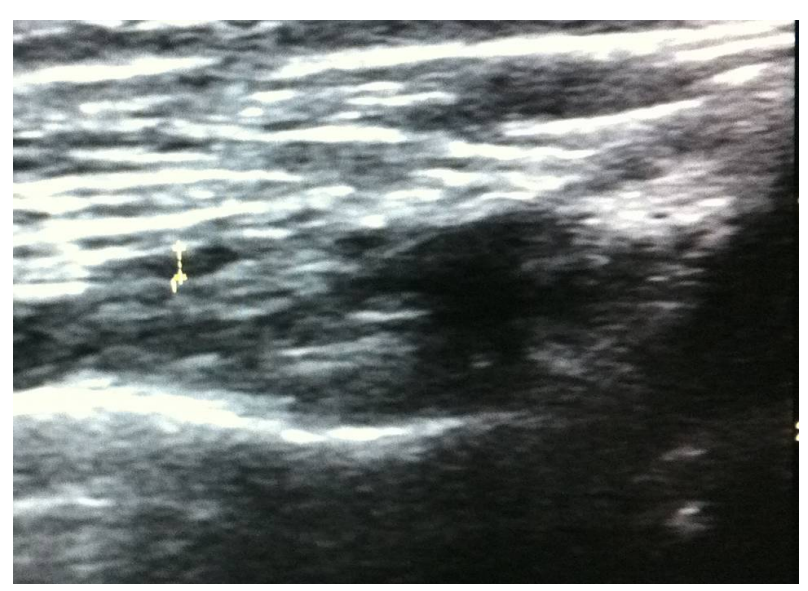

Fig. 4. Acute accumulation of liquid in soft tissues of forearm, caused by cytostatic extravasation

The quantitative changes of venous blood stream on the background of chemotherapy according to the data of dopplerography and duplex scanning (Table 1).

\section{Table 1}

Quantitative parameters of dopplerography and duplex scanning at using peripheral veins of forearm for chemotherapy $(n=12)$

\begin{tabular}{ccc}
\hline Parameters of blood stream & Initial parameters & In $\mathbf{6}$ months \\
\hline AEVC, $\mathrm{mm}$ & $3,1 \pm 0,4$ & $1,9 \pm 0,9$ \\
TAmx $1, \mathrm{~cm} / \mathrm{s}$ & $6,4 \pm 2,4$ & Was not determined \\
$\mathrm{FV} 1, \mathrm{l} / \mathrm{min}$ & $0,053 \pm 0,012$ & Was not determined
\end{tabular}

The index of stricture formation of exploited vein exceeded $80 \%$ in all observations. 


\section{Discussion of research results}

The changes in veins in the process of chemotherapy were of the same type in all patients and usually took place after four chemotherapy courses.

The intensity of revealed changes was influenced by the treatment duration, character of cytostatic (they differed by the mechanism of local lesion - of irritating or vesicant effect) and also by the number of technical complications, connected with vein puncture during the procedure.

The duration of peripheral veins exploitation was not influenced by sex, age and main diagnosis (localization of primary tumor) of patients.

In all patients the infusion of cytostatics in peripheral vein led to the chemical lesion of venous tubes of certain intensity. The clinical manifestations of injuring effect of cytostatics varied from inflammatory reaction, pain edema, temperature rise, arythema, phlebitis, sclerosis and pigmentation in vein zone to chemical cellulite, induration and also desquamation of skin, bubbles, severe lesion of tissues and necrosis in patients with extravasation of preparations.

Extravasation of cytostatics always led to the limitation of extremity functions, development of septic state, disorder of antitumor therapy protocols and worsening of patient's life quality.

The adequately functioning vascular access was considered as the one that corresponded to the main requirements: the method of its exploitation and manipulation is maximally safe for patient, repeated use and long-term application is necessary for realization of the whole planned program of treatment without creation of new access.

None peripheral access corresponded to such requirements in observations that excluded a possibility of their exploitation in the context of realization of the whole long-term program of antitumor treatment.

\section{Conclusions}

1. At exploitation of forearm peripheral veins for antitumor therapy, the functional and morphological lesions take place in them fast that is a result of the direct cytotoxic action of preparations on endothelial cells of internal tunic of vein.

2. These changes are revealed as thickening of forearm vein wall not only in the place of its puncture but on essential extent in the direction to cephalic vein, decrease of elasticity of vein walls and diminution of its lumen diameter.

3. The organized hematomas with calcification appear in soft tissues in the places of punctures of vein and paravasaly. The bloodstream in forearm veins is absent.

4. Such inadequacy of peripheral venous channel makes impossible the continuation of chemotherapy through forearm veins.

5. Prognostication of such complication needs creation of alternative venous access (implantation of vascular port in central vein or formation of arteriovenous fistula) at planning of the system antitumor therapy at the beginning of disease.

\section{References}

[1] Nass, S. J., Moses, H. L., Mendelsohn, J. (Eds.) (2010). A National Cancer Clinical Trials Systemforthe 21 st Century: Reinvigoratingthe NCIC ooperative GroupProgram. Washington: National Academies Press, 316. doi: 10.17226/12879

[2] Baer, A. R., Kelly, C. A., Bruinooge, S. S., Runowicz, C. D., Blayney, D. W. (2010). Challenges to National Cancer Institute-Supported Cooperative Group Clinical Trial Participation: An ASCO Survey of Cooperative Group Sites. Journal of Oncology Practice, 6 (3), 114-117. doi: 10.1200/jop.200028

[3] Infusion Nurses Society (2011). Infusion Nursing Standards of Practice. Journal of Infusion Nursing, 34 (1S).

[4] Webster, J., Clarke, S., Paterson, D., Hutton, A., Dyk, S. V., Gale, C., Hopkins, T. (2008). Routine care of peripheral intravenous catheters versus clinically indicated replacement: randomised controlled trial. BMJ, 337 (1), a337-a339. doi: 10.1136/bmj.a339

[5] Keuter, X. H. A., De Smet, A. A. E. A., Kessels, A. G. H., van der Sande, F. M., Welten, R. J. T. J., Tordoir, J. H. M. (2008). A randomized multicenter study of the outcome of brachial-basilic arteriovenous fis- 
tula and prosthetic brachial-antecubital forearm loop as vascular access for hemodialysis. Journal of Vascular Surgery, 47 (2), 395-401. doi: 10.1016/j.jvs.2007.09.063

[6] Field, K., McFarlane, C., Cheng, A. C., Hughes, A. J., Jacobs, E., Styles, K. et. al. (2007). Incidence of Catheter-Related Bloodstream Infection Among Patients With a Needleless, Mechanical ValveBased Intravenous Connector in an Australian Hematology-Oncology Unit. Infection Control \& Hospital Epidemiology, 28 (5), 610-613. doi: 10.1086/516660

[7] Bikkulova, D. Sh. (2014). Clinical recommendations for venous access. Russian Journal of Pediatric Hematology and Oncology, 3, 73-77.

[8] Koksoy, C., Kuzu, A., Kutlay, J., Erden, I., Ozcan, H., Ergin, K. (1995). The diagnostic value of colour doppler ultrasound in central venous catheter related thrombosis. Clinical Radiology, 50 (10), 687-689. doi: 10.1016/s0009-9260(05)83313-6

[9] Cassidy, J., Bissett, D., Spence, R., Payne, M. (2010). Oxford handbook of oncology. New York: Oxford University Press, 831.

[10] Ferring, M., Claridge, M., Smith, S. A., Wilmink, T. (2010). Routine Preoperative Vascular Ultrasound Improves Patency and Use of Arteriovenous Fistulas for Hemodialysis: A Randomized Trial. Clinical Journal of the American Society of Nephrology, 5 (12), 2236-2244. doi: 10.2215/cjn.02820310

[11] Nassaji-Zavareh, M., Ghorbani, R. (2007). Peripheral intravenous catheter related phlebitis and related risk factors. Singapore Medical Journal, 48 (8), 733-736.

[12] Ferring, M., Henderson, J., Wilmink, A., Smith, S. (2008). Vascular ultrasound for the pre-operative evaluation prior to arteriovenous fistula formation for haemodialysis: review of the evidence. Nephrology Dialysis Transplantation, 23 (6), 1809-1815. doi: 10.1093/ndt/gfn001

[13] Salgado, C. D., Chinnes, L., Paczesny, T. H., Cantey, J. R. (2007). Increased Rate of Catheter-Related Bloodstream Infection Associated With Use of a Needleless Mechanical Valve Device at a Long-Term Acute Care Hospital. Infection Control \& Hospital Epidemiology, 28 (6), 684-688. doi: 10.1086/516800

[14] Rupp, M. E., Sholtz, L. A., Jourdan, D. R., Marion, N. D., Tyner, L. K., Fey, P. D. et. al. (2007). Outbreak of Bloodstream Infection Temporally Associated with the Use of an Intravascular Needleless Valve. Clinical Infectious Diseases, 44 (11), 1408-1414. doi: 10.1086/517538

[15] Lee, T., Barker, J., Allon, M. (2007). Comparison of Survival of Upper Arm Arteriovenous Fistulas and Grafts after Failed Forearm Fistula. Journal of the American Society of Nephrology, 18 (6), 1936-1941. doi: 10.1681/asn.2006101119

[16] Shifris, I. M. (2011). Infections of vascular access. Ukrainian Journal of Nephrology and Dialysis, 3, 68-74.

[17] Catney, M. R., Hillis, S., Wakefield, B., Simpson, L., Domino, L., Keller, S. et. al. (2001). Relationship Between Peripheral Intravenous Catheter Dwell Time and the Development of Phlebitis and Infiltration. Journal of Infusion Nursing, 24 (5), 332-341. doi: 10.1097/00129804-200109000-00008

[18] Frey, A. M., Schears, G. J. (2006). Why Are We Stuck on Tape and Suture? Journal of Infusion Nursing, 29 (1), 34-38. doi: 10.1097/00129804-200601000-00007

[19] Ener, R. A., Meglathery, S. B., Styler, M. (2004). Extravasation of systemic hemato-oncological therapies. Annals of Oncology, 15 (6), 858-862. doi: 10.1093/annonc/mdh214

[20] Baarslag, H. J., van Beek, E. J. R., Koopman, M. M. W., Reekers, J. A. (2002). Prospective Study of Color Duplex Ultrasonography Compared with Contrast Venography in Patients Suspected of Having Deep Venous Thrombosis of the Upper Extremities. Annals of Internal Medicine, 136 (12), 865. doi: 10.7326/00034819-136-12-200206180-00007 